\title{
Pericardial Injury With Cardiac Tamponade From Multiple Stab Injuries of the Trunk: Incidental Release of Cardiac Tamponade
}

\author{
Pil Young Jung ${ }^{1}$, Kwan Wook Kim² \\ 'Department of Surgery, Yonsei university Wonju college of medicine, Wonju Severance Christian Hospital \\ ${ }^{2}$ Trauma center, Department of Thoracic and Cardiovascular Surgery, CHA University, CHA Bundang Medical Center
}

Traumatic hemopericardium with cardiac tamponade is a rare but life-threatening condition. We report the successful treatment of hemopericardium with cardiac tamponade caused by multiple stab injuries of the trunk.

Key Words: Hemopericardium, Cardiac tamponade

(Trauma Image Proced 2019(1):22-24)

\section{CASE}

From a regional local hospital, a 34-year-old man who had schizophrenia was transferred to our institution with multiple stab injuries of the trunk, sustained in a suicide attempt (Fig. 1.). At admission, the patient's vital signs were unstable; cardiac arrest occurred, and return of spontaneous circulation was achieved after one cycle of cardiopulmonary resuscitation. The extended focused assessment sonography in trauma revealed positive signs in the pericardium and splenorenal space. Computed tomographic scans taken at the previous hospital showed hemopericardium and hemoperitoneum (Fig. 2.). Emergency surgery of the chest and abdomen was begun simultaneously. During opening of the chest with median sternotomy, cardiac arrest occurred again; We performed open cardiac massage, and spontaneous circulation resumed. The surgical finding was a complete transec- tion of the left internal mammary vessels, which was the cause of hemopericardium (Fig. 3.). The epicardium of the right ventricle, the greater omentum, and the spleen were injured; the diaphragmatic injury may have served as a pericardial window of injury into the abdomen cavity. We performed ligation of the left internal mammary vessels, splenectomy, omentectomy, and primary repair of the diaphragm. The patient recovered and was discharged without any complication 24 days after admission (Fig. 4.).

\section{DISCUSSION}

Penetrating cardiac injuries have been described since ancient times and are still considered a challenge in trauma surgery because of their lethality and the urgency of treatment (1). Penetrating chest trauma is one of the most dangerous injuries incurred by patients, and

Received: February 3, 2019 Revised: April 9, 2019 Accepted: April 16, 2019

Correspondence to: Pil Young Jung, Department of Surgery, Yonsei university Wonju college of medicine, Wonju Severance Christian Hospital, Trauma center, 220-701, 20 Ilsan-ro, Wonju-si, Gangwon-do, South Korea

Tel: 82-33-741-0882, Fax: 82-33-741-0574, E-mail: surgery4trauma@yonsei.ac.kr

Copyright (c) 2019 Korean Association for Research, Procedures and Education on Trauma. All rights reserved.

@This is an open-access article distributed under the terms of the Creative Commons Attribution Non-Commercial License (http://creativecommons.org/ licenses/by-nc/4.0) which permits unrestricted noncommercial use, distribution, and reproduction in any medium, provided the original work is properly cited 


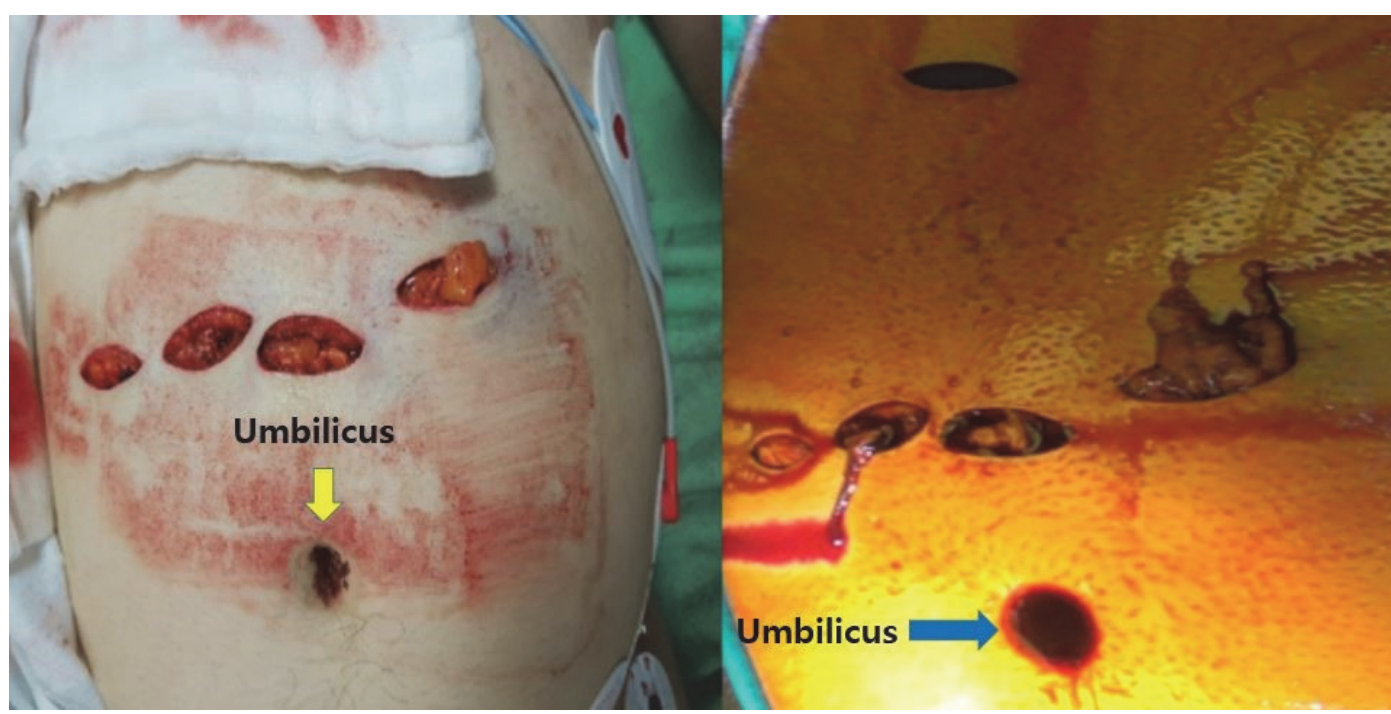

Fig. 1. Multiple stab injuries (five sites) on the patient's trunk.

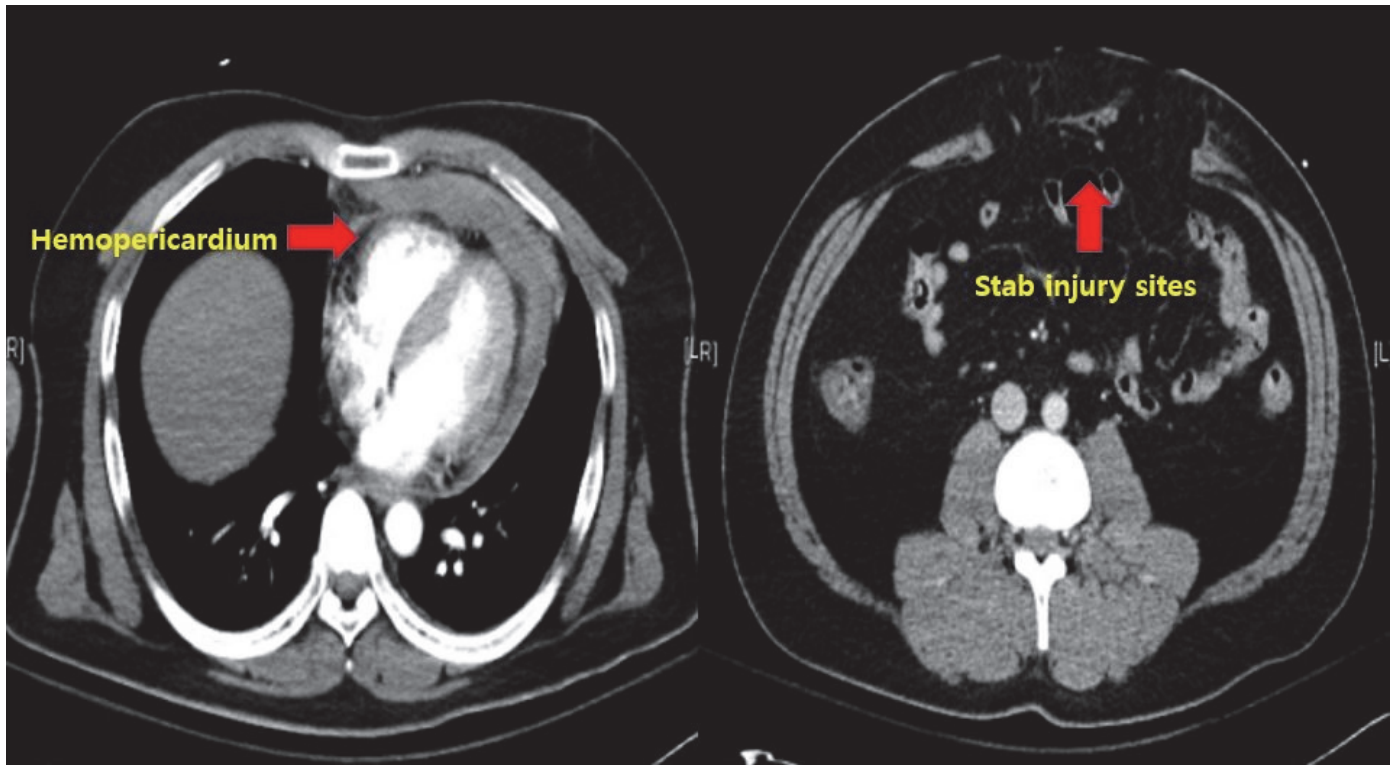

Fig. 2. Computed tomographic scan, showing hemopericardium and abdominal injury.

penetrating cardiac injuries are among the most lethal. Because of the life-threatening physiologic changes caused by these injuries, including pericardial tamponade and hemorrhagic shock, management of penetrating cardiac injuries has focused on rapid assessment and therapeutic maneuvers (2). In our patient, pericardial injury with cardiac tamponade was caused by multiple stab injuries. In this case, the diaphragm injury served as a pericardial window to the abdominal cavity; however, the patient survived, despite major bleeding and the relatively long time for transfer between hospitals.

\section{Conflicts of Interest Statement}

None of authors have a conflict of interest.

\section{REFERENCE}

1. Pereira BM, Nogueira VB, Calderan TR, et al. Penetrating 


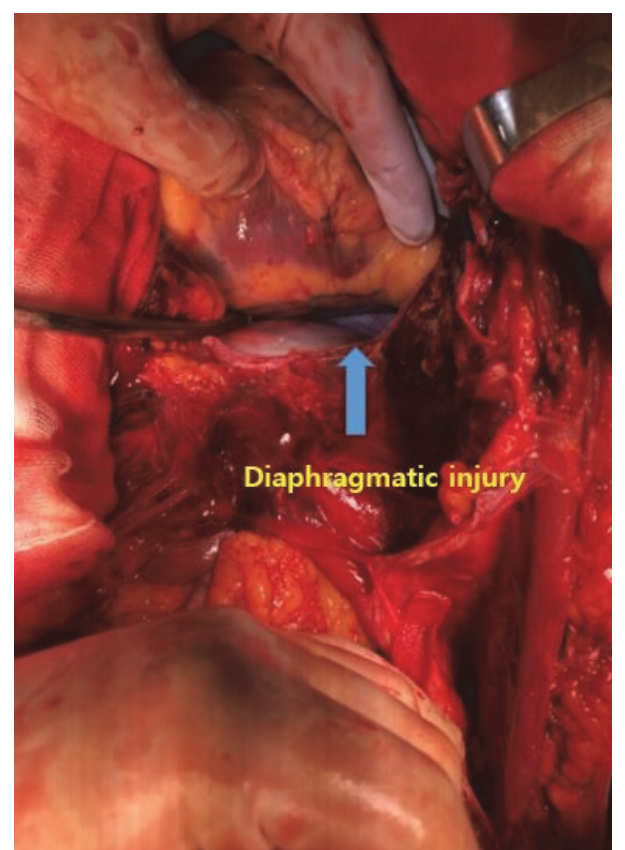

Fig. 3. Diaphragm injury caused an opening into the abdominal cavity, which served as a pericardial window.

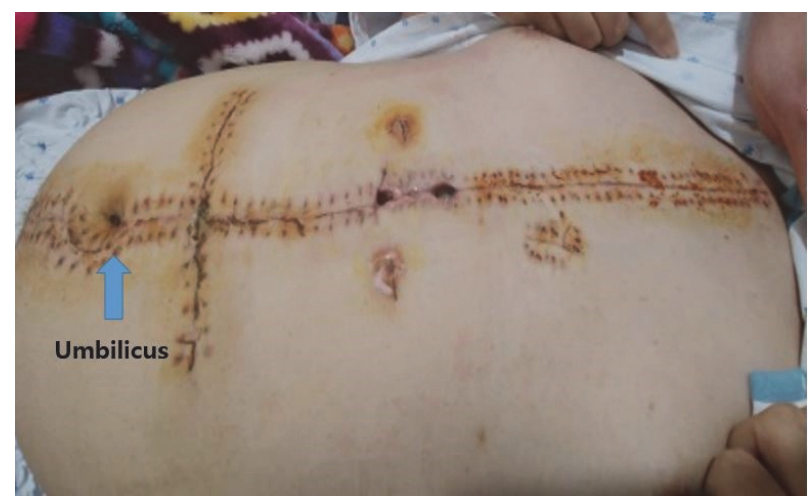

Fig. 4. The postoperative wound.

cardiac trauma: 20-y experience from a university teaching hospital. J Surg Res 2013;183:792 - 7.

2. Chestovich PJ1, McNicoll CF1, Fraser DR1, et al. Selective use of pericardial window and drainage as sole treatment for hemopericardium from penetrating chest trauma. Trauma Surg Acute Care Open. 2018 Aug 30;3(1) 